\title{
Five-Point Two-Loop Amplitudes from Numerical Unitarity
}

\author{
Samuel Abreu, ${ }^{a}$ Fernando Febres Cordero*, ${ }^{*}$ Harald Ita, ${ }^{a}$ Ben Page $\dagger,{ }^{a}$ and \\ Mao Zeng ${ }^{b}$ \\ ${ }^{a}$ Physikalisches Institut, Albert-Ludwigs-Universität, Freiburg \\ D-79104 Freibug, Germany \\ ${ }^{b}$ Mani L. Bhaumik Institute for Theoretical Physics \\ UCLA Department of Physics and Astronomy \\ Los Angeles, CA 90095, USA \\ E-mail: ffebres@physik.uni-freiburg.de, \\ ben.page@physik.uni-freiburg.de
}

\begin{abstract}
We present advances in the development of the numerical unitarity method for the computation of multi-loop amplitudes in QCD. As an application, we show results for all the leading-color twoloop five-gluon helicity amplitudes. The amplitudes are reduced to a linear combination of master integrals by employing unitarity-compatible integration-by-parts identities, and the corresponding integral coefficients are computed in an exact manner on rational phase-space points through finite fields arithmetics.
\end{abstract}

Loops and Legs in Quantum Field Theory (LL2018)

29 April 2018 - 04 May 2018

St. Goar, Germany

\footnotetext{
${ }^{*}$ Speaker.

${ }^{\dagger}$ Speaker.
} 


\section{Introduction}

In the decade to come the LHC will be testing the Standard Model of particle physics (SM) to an unprecedented level of accuracy. This provides a natural pressure for innovation within the theory community, as theoretical uncertainties associated to a large amount of observables will need to be reduced to the few-percent level. To achieve this, both next-to-next-to-leading-order (NNLO) QCD corrections and next-to-leading-order (NLO) electroweak corrections will need to be computed for processes involving many scales. Though our capacity to perform the latter type of corrections has matured over the last few years, the former stills present a variety of challenges. NNLO QCD corrections are nevertheless highly desirable - relevant processes include 3-jet, $H+2$ jet, $V+2$-jet, $t \bar{t}+$ jet production (for an extended discussion see ref. [1]). For example, the importance of NNLO QCD corrections to $W$ production with two $b$ jets has been recently highlighted in ref. [2] where an analysis based on exclusive sums shows that the $\mathscr{O}\left(\alpha_{s}^{2}\right)$ corrections can be sizable and necessary to stabilize the perturbative series of associated observables, which are relevant for ongoing and future studies of the $b$-quark Yukawa coupling (see for example ref. [3, 4]).

NNLO QCD corrections require many ingredients. Among the most complicated is constructing a procedure to handle the different IR-divergent contributions in such a way that the multiple phase-space integrations required can be achieved numerically. Over the last 15 years a number of IR subtraction schemes have been developed, which explicitly manifest the cancellation of IR divergences and are starting to show great potential for automation. Among them are antenna subtraction [5, 6], sector improved residue subtraction [7], $q_{T}$ slicing [8] and $N$-jettiness slicing [9, 10].

Another challenging ingredient which is the focus of these talks, is the computation of twoloop amplitudes with a higher number of scales. The first calculation of a two-loop five-gluon amplitude appeared five years ago, for the special case of all positive helicities [11]. Since then, a number of advances have been made for this particular amplitude [12, 13, 14], followed by further calculations of the same helicity configuration at higher multiplicity $[15,16]$. More recently the complete set of helicity configurations were also studied with a combination of numeric and analytic techniques [17], and a follow up including a full reduction to master integrals was presented at this conference. ${ }^{1}$ We also highlight the recent appearance of advanced integration-by-parts reductions for massless two-loop five-point amplitudes of refs. [18, 19]. Related promising techniques for handling multi-scale two-loop problems have been presented at this conference. ${ }^{2}$

In principle, these complex amplitude calculations can be performed by constructing the full set of associated two-loop Feynman diagrams. However, as is well known, the complexity of computing high-multiplicity processes in this way leads to very large intermediate expressions in analytic implementations (even at one loop). In this talk, we focus on an approach [20, 21] that exploits the unitarity method $[22,23,24,25]$ in order to numerically compute multi-loop amplitudes directly from gauge-invariant building blocks. The method makes use of an advanced decomposition of the amplitude's integrand based on master integrands and surface terms, the latter being constructed from unitarity-compatible integration-by-parts identities. With the master integrals available, ${ }^{3}$ we can then obtain the desired amplitudes. As an application, we show a

\footnotetext{
${ }^{1}$ See the talk by S. Badger in these proceedings PoS (LL2018) 006.

${ }^{2}$ See the talks of W. Torres Bobadilla PoS (LL2018) 036 and K. Larsen PoS (LL2018) 064.

${ }^{3}$ See the talks on these proceedings by J. Henn PoS (LL2018) 014 and C. Papadopoulos PoS (LL2018) 015.
} 
computation of the leading-color two-loop five-gluon helicity amplitudes.

The remainder of these proceedings is divided into three sections. In section 2 we describe details of our computational framework. Section 3 shows our results for the five-gluon helicity amplitudes, and finally we conclude in section 4 with an outlook about the prospects of the developed techniques.

\section{Numerical unitarity}

In order to perform an amplitude calculation with numerical unitarity we begin by classifying all propagator structures that can appear in the integrand of the amplitude. These structures are represented by a set of diagrams $\Delta=\left\{\Gamma_{i}\right\}$, which naturally can be organized in a hierarchical way. As an illustrative example, we show in figure 1 the full hierarchy of propagator structures that appear for planar two-loop four-point massless amplitudes. The top row contains all maximal diagrams, those with seven propagators, and we go down to the bottom of the hierarchy to the three-propagator diagram, the so called sunrise diagram.
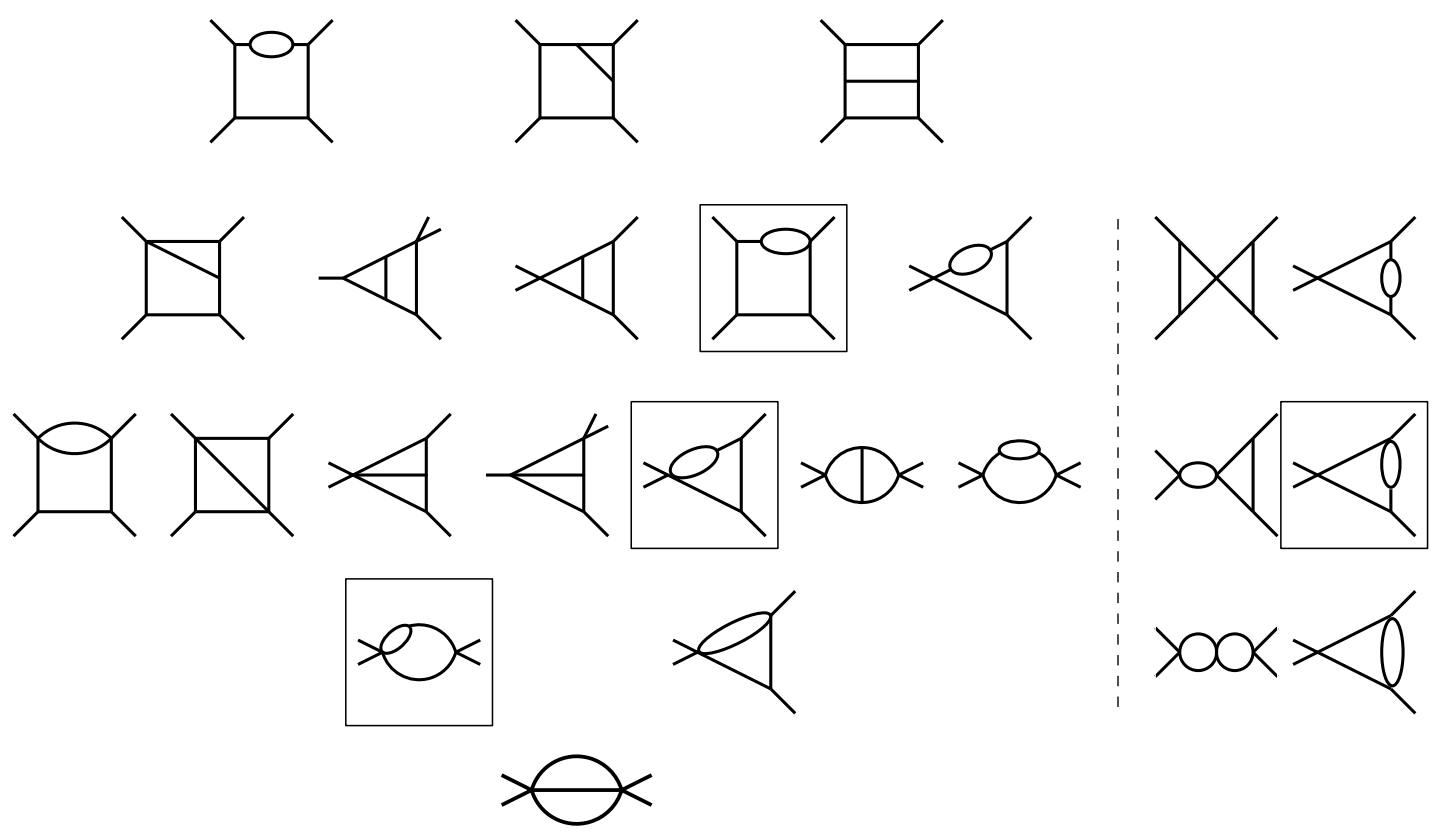

Figure 1: The planar hierarchy of propagator structures in a $2 \rightarrow 2$ massless amplitude. All edges represent massless propagators. We include topologically inequivalent diagrams only. We highlight with boxes the diagrams that do not have associated products of trees. To the left of the dashed line we collect the subhierarchy of propagator structures that have the sunrise as their minimal cut.

To compute the amplitude, we start from the standard decomposition of an amplitude in terms of master integrals:

$$
\mathscr{A}^{(L)}=\sum_{\Gamma \in \Delta} \sum_{i \in M_{\Gamma}} c_{\Gamma, i} \mathscr{I}_{\Gamma, i}
$$


where $L$ is the number of loops, and $M_{\Gamma}$ represents the set of master integrals associated to $\Gamma$ (notice that it can be empty for many of the diagrams in $\Delta$ ). The process-dependent coefficients $c_{\Gamma, i}$ are to be determined. We note that they depend on the dimensional regularization parameters $D$ and $D_{s}$.

Following a similar approach to the one-loop numerical unitarity method [26, 27, 28, 29], we promote the previous ansatz to the integrand level. That is, denoting the $L$-loop integrand by $\mathscr{A}^{(L)}\left(\ell_{l}\right)$, with $\ell_{l}=\ell_{1}, \ldots, \ell_{L}$, we write:

$$
\mathscr{A}^{(k)}\left(\ell_{l}\right)=\sum_{\Gamma \in \Delta} \sum_{i \in M_{\Gamma} \cup S_{\Gamma}} \frac{c_{\Gamma, i} m_{\Gamma, i}\left(\ell_{l}\right)}{\prod_{j \in P_{\Gamma}} \rho_{j}},
$$

where $\rho_{j}$ are the inverse propagators and $P_{\Gamma}$ represents the set of propagators in diagram $\Gamma$. The $m_{\Gamma, i}$ functions form a basis parametrizing the integrand associated to the diagram $\Gamma$ (up to corresponding power counting). In order for the coefficients of the integrand level masters to correspond to those in eq. (2.1), the additional terms (with $i \in S_{\Gamma}$ ) must integrate to zero, and hence we call them surface terms. We stress that the $m_{\Gamma, i}\left(\ell_{l}\right)$ functions depend only on the kinematics of the process, and not on the spin or helicity of the scattering states.

The ansatz (2.2) holds for arbitrary loop momenta $\ell_{l}$, and one can exploit this to construct linear systems of equations from which one can compute all the coefficients $c_{\Gamma, i}$. In generalized unitarity calculations, compact systems of equations can be constructed by taking residues around the multi-propagator poles corresponding to each diagram $\Gamma$,

$$
\sum_{\text {states }} \prod_{i \in T_{\Gamma}} \mathscr{A}_{i}^{\text {tree }}\left(\ell_{l}^{\Gamma}\right)=\sum_{\substack{\Gamma^{\prime} \geq \Gamma \\ i \in M_{\Gamma^{\prime}} \cup S_{\Gamma^{\prime}}}} \frac{c_{\Gamma^{\prime}, i} m_{\Gamma^{\prime}, i}\left(\ell_{l}^{\Gamma}\right)}{\prod_{j \in P_{j}} \rho_{j}} .
$$

On such a residue, the integrand factorizes into a product of trees. The relations obtained from eq. (2.3) are called cut equations. We note that although starting at two loops certain diagrams do not have a corresponding product of trees (e.g. the boxed ones in figure 1), following the procedure described in ref. [30] one can always find enough cut equations to solve for all coefficients.

\section{Master-surface decompositions}

The key component in writing down the integrand ansatz (2.2) is a complete set of surface terms for each set of propagator structures $\Gamma$. Precisely, one needs to construct the full set of $m_{\Gamma, i}$ which satisfy

$$
0=\int\left[d^{D} \ell_{l}\right] \frac{m_{\Gamma, i}}{\prod_{j \in P_{\Gamma}} \rho_{j}}, \quad \text { with } \quad\left[d^{D} \ell_{l}\right] \equiv \prod_{l=1}^{L} \mathrm{~d}^{D} \ell_{l},
$$

where the $m_{\Gamma, i}$ are naturally polynomials in the loop momenta. The construction of such a set of surface terms follows the description in [20, 21, 31]. We start by writing the integration-by-parts (IBP) relation,

$$
\int\left[d^{D} \ell_{l}\right] \sum_{k} \frac{\partial}{\partial \ell_{k}^{v}}\left[\frac{u_{k}^{v}}{\prod_{j \in P_{\Gamma}} \rho_{j}}\right]=0 .
$$

We note that the differential operator in eq. (2.5) will in general generate terms with higher propagator power. In order to account for this mismatch between eqs. (2.4) and (2.5) we choose vectors 
$u_{k}^{v}$ that preserve the propagator structure associated to $\Gamma$. This is ensured by defining the so called IBP-generating vectors [32] according to:

$$
u_{k}^{v} \frac{\partial}{\partial \ell_{k}^{v}} \rho_{j}=f_{j} \rho_{j}, \quad \forall j \in P_{\Gamma}
$$

where $1 \leq k \leq L$ and there is no sum over $j$. We require the $f_{j}$ functions to be polynomials in the loop momentum components, which we parametrize with a convenient set of variables.

To find the necessary IBP-generating vectors, we start from the ansatz

$$
u_{k}^{v} \frac{\partial}{\partial \ell_{k}^{v}}=\left(u_{k a}^{\text {loop }} \ell_{a}^{v}+u_{k c}^{\text {ext }} p_{c}^{v}\right) \frac{\partial}{\partial \ell_{k}^{v}}
$$

where the labels $a$ and $c$ are summed over. Then eq. (2.6) becomes [21]:

$$
\left(u_{k a}^{\text {loop }} \ell_{a}^{v}+u_{k c}^{\text {ext }} p_{c}^{v}\right) \frac{\partial}{\partial \ell_{k}^{v}}\left(\begin{array}{c}
\rho_{j(1)} \\
\rho_{j(2)} \\
\vdots \\
\rho_{j(|\Gamma|)}
\end{array}\right)-\left(\begin{array}{c}
f_{j(1)} \rho_{j(1)} \\
f_{j(2)} \rho_{j(2)} \\
\vdots \\
f_{j(|\Gamma|)} \rho_{j(|\Gamma|)}
\end{array}\right)=0 .
$$

This is a polynomial relation, known as a syzygy equation, which can be solved using algorithms from computational algebraic geometry. We use the software Singular [33] to find solutions for all required diagrams in our calculations. Analytic solutions can be found in under a second for the most complicated case necessary for the results in section 3 .

As a simple example of a surface term let us consider this procedure in the case of the one-loop one-mass triangle diagram with propagators and external kinematics given by

$$
\begin{aligned}
\rho_{1}=\left(\ell+p_{1}\right)^{2}, \quad \rho_{2}=\ell^{2}, \quad \rho_{3} & =\left(\ell-p_{2}\right)^{2}, \\
p_{1}^{2}=p_{2}^{2}=0, \quad\left(p_{1}+p_{2}\right)^{2} & =s .
\end{aligned}
$$

We find that there is a single generating vector,

$$
\begin{aligned}
& u^{v}=u_{1}^{\text {ext }} p_{1}^{v}+u_{2}^{\text {ext }} p_{2}^{v}+u^{\text {loop }} \ell^{v} \\
& u^{v}=\left(\rho_{3}-\rho_{2}\right) p_{1}^{v}+\left(\rho_{1}+\rho_{2}\right) p_{2}^{v}+\left(-s+2 \rho_{3}-2 \rho_{2}\right) \ell^{v} .
\end{aligned}
$$

As this satisfies eq. (2.6), the result of inserting this expression into eq. (2.5) produces a result with no raised propagator powers,

$$
0=\int \mathrm{d}^{D} \ell \frac{\partial}{\partial l^{v}} \frac{u^{v}}{\rho_{1} \rho_{2} \rho_{3}}=\int \mathrm{d}^{D} \ell \frac{1}{\rho_{1} \rho_{2} \rho_{3}}\left[-(D-4) s-2(D-3) \rho_{2}+2(D-3) \rho_{3}\right] .
$$

This relation can then be used either as a surface term, or solved to obtain the well known IBP relation that relates the scalar triangle to the scalar bubble. Techniques for analytically solving a system of surface terms to generate IBP relations are explored in [34].

Once a set of IBP-generating vectors is produced for a given diagram, a full decomposition $\left\{m_{\Gamma, i}\right\}$ is achieved by multiplying them with a complete set of irreducible numerators respecting power counting [31], up until the dimension of the space $S_{\Gamma}$ is filled. The complement of the space can then be filled with independent insertions to produce the corresponding master integrands in $M_{\Gamma}$. The full decomposition of all planar five-point massless amplitudes in $\mathrm{C}++$ code takes about $3.5 \mathrm{MB}$ of data, and we expect it to be possible to construct even more compact expressions in the future. 


\section{Numerical unitarity and finite fields}

In recent years it has been demonstrated that so-called "finite fields" can aid in the calculation of multi-loop scattering amplitudes [35, 36]. By exploiting their mathematical structure, one can use computer integers to obtain exact results in calculations involving only field operations. In contrast to more commonly seen floating-point numerics, it is not possible to solve arbitrary polynomial equations and this presents challenges for constructing a numerical unitarity approach, many of which were solved in [36].

In the numerical unitarity method it is necessary to use loop momenta satisfying the on-shell conditions associated to each of the propagator structures $\Gamma$. As these are a system of simultaneous quadratic equations this is a non-trivial exercise when employing finite fields. To parametrize these "on-shell phase spaces" we employ an adaptation of the van Neerven-Vermaseren basis [37]. We begin by parametrizing the loop momentum of each strand in the given diagram $\ell_{l}$ (with $l=1,2$ for all factorized (one-loop squared) diagrams and $l=1,2,3$ otherwise) as [31, 20, 21]:

$$
\begin{aligned}
\ell_{l} & =\sum_{j \in B_{l}^{p}} v_{l}^{j} r^{l j}+\sum_{j \in B_{l}^{t}} v_{l}^{j} \alpha^{l j}+\sum_{i \in B^{c t}} \frac{n^{i}}{\left(n^{i}\right)^{2}} \alpha^{l i}+\sum_{i \in B^{\varepsilon}} n^{i} \mu_{l}^{i}, \\
r^{l j} & =-\frac{1}{2}\left(\rho_{l j}-\left(q_{l j}\right)^{2}-\rho_{l(j-1)}+\left(q_{l(j-1)}\right)^{2}\right), \\
\mu_{l l} & \equiv \mu_{l} \cdot \mu_{l}=\rho_{l 0}-\sum_{v=0}^{3} \ell_{l}^{v} \ell_{l v},
\end{aligned}
$$

where the index sets $B_{l}^{p}, B_{l}^{t}, B^{c t}$ and $B^{\varepsilon}$ have the following physical interpretation: $B_{l}^{p}$ is the physical space spanned by the momenta attached to the strand $l ; B^{c t}$ is the physical (4-D) common transverse space (transverse to all $B_{l}^{p}$ 's); $B_{l}^{t}$ is the transverse space to $B_{l}^{p}$ not overlapping $B^{c t}$; and $B^{\varepsilon}$ is the extra dimensional $D-4$ space. The $q_{l j}$ are linear combinations of external momenta. The vectors $v_{l}^{j}$ are a basis of the physical scattering plane chosen to be dual to the external momenta, and the $n^{i}$ are an orthogonal basis of the space transverse to the physical scattering space.

In this coordinate system, it is trivial to satisfy the on-shell conditions by setting the associated propagator variables $\rho$ to 0 . Further, it is clear that for any finite field valued choice of the irreducible scalar products $\alpha$, the 4-dimensional components of the loop momenta also belong to the finite field. In order to treat the typically algebraic valued $(D-4)$ dimensional components of the loop momenta, $\mu_{i}$, we represent them abstractly, taking the $\mu_{i}$ themselves as basis vectors,

$$
\ell_{l}^{(D-4)}=w_{l, 1} \mu_{1}+w_{l, 2} \mu_{2} .
$$

This choice must then be respected by the scalar product, that is

$$
\ell_{r} \cdot \ell_{s}=\ell_{r}^{4} \cdot \ell_{s}^{4}-\sum_{i, j=1}^{2} w_{i}^{r} w_{j}^{s} \mu_{i j}
$$

A further complexity when performing unitarity-based computation in finite fields, is the handling of the internal states that appear on a cut propagator. In these states, normalization factors appear that cannot be represented in the finite field. Instead of state sums, we insert a light-cone projector operator in the $D_{s}$-dimensional space of the polarization vectors

$$
P_{l}^{\mu v}=-g^{\mu v}+\frac{\ell_{l}^{\mu} \eta^{v}+\eta^{\mu} \ell_{l}^{v}}{\eta \cdot \ell_{l}}
$$


where $\eta$ is an arbitrary light-like reference vector with rational components (which can of course be represented in the finite field) and that must fulfil $\eta \cdot \ell_{l} \neq 0$ and $\eta \cdot \mu_{i}=0$. Although this is a sound solution, we have also implemented modified physical states for performing the calculations as it is more efficient for larger values of $D_{s}$. In this way, intermediate square roots can be avoided in a gluonic numerical unitarity calculation. More details are given in [21].

\section{The planar two-loop five-gluon helicity amplitudes}

The planar diagram hierarchy for two-loop five-point massless amplitudes is comprised of 60 topologically independent diagrams, in which 25 master integrals appear. In figure 2 we show the subset of diagrams with master integrals and their corresponding multiplicity. In total, considering all permutations of the external legs maintaining the cyclic order, we need to compute 155 integral coefficients.
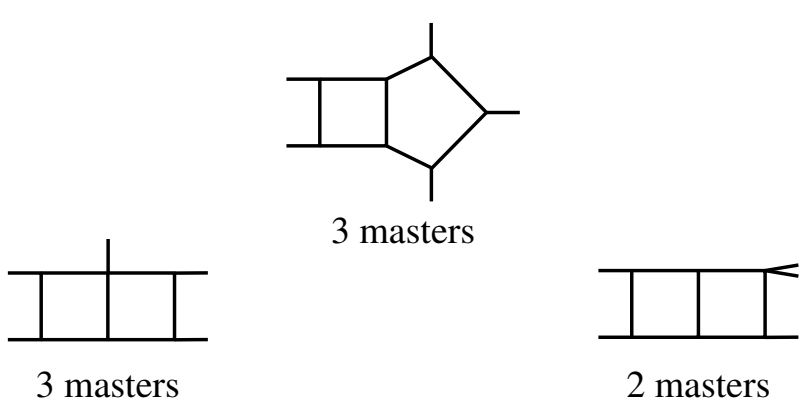
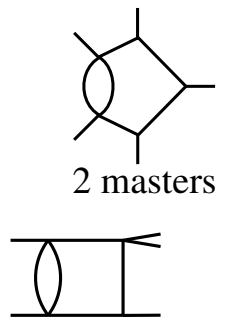

1 master

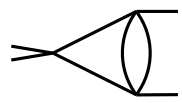

1 master
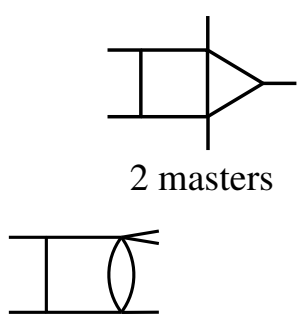

1 master
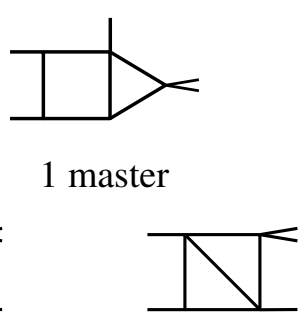

2 masters

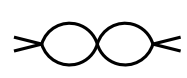

1 master

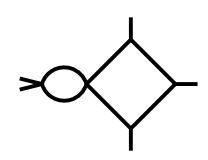

1 master

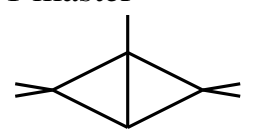

1 master

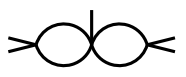

1 master

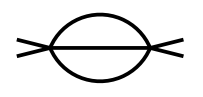

1 master

Figure 2: Propagator structures with master integrals for massless two-loop five-point amplitudes.

We present results for the four independent helicity configurations (up to cyclic permutations and parity transformations) that appear for the scattering of five gluons. We include all-plus and single-minus helicity configurations, though they are not necessary for a potential NNLO QCD 
calculation of three-jet production, as their corresponding tree-level amplitudes vanish. The results we present for the amplitudes are obtained in the Euclidean phase-space point

$$
\begin{aligned}
& p_{1}=\left(\frac{1}{2}, \frac{1}{16}, \frac{i}{16}, \frac{1}{2}\right), \quad p_{2}=\left(-\frac{1}{2}, 0,0, \frac{1}{2}\right), \quad p_{3}=\left(\frac{9}{2},-\frac{9}{2}, \frac{7 i}{2}, \frac{7}{2}\right), \\
& p_{4}=\left(-\frac{23}{4}, \frac{61}{16},-\frac{131 i}{16},-\frac{37}{4}\right), \quad p_{5}=\left(\frac{5}{4}, \frac{5}{8}, \frac{37 i}{8}, \frac{19}{4}\right),
\end{aligned}
$$

with the corresponding invariants $s_{12}=-1, s_{23}=-8, s_{34}=-10, s_{45}=-7, s_{51}=-3$. We set the renormalization scale $\mu$ to $\mu=1$. We present results in the 't Hooft-Veltman scheme for dimensional regularization [38]. As our coefficients have been computed in an exact manner, the numerical precision of our results is only limited by the precision to which the analytic expressions of the integrals are computed $[39,40]$. For the evaluation of the generalized polylogarithms we employ GiNaC [41].

\begin{tabular}{|c|c|c|c|c|c|}
\hline $\mathscr{A}^{(2)} / \mathscr{A}^{\text {(norm) }}$ & $\varepsilon^{-4}$ & $\varepsilon^{-3}$ & $\varepsilon^{-2}$ & $\varepsilon^{-1}$ & $\varepsilon^{0}$ \\
\hline$\left(1^{+}, 2^{+}, 3^{+}, 4^{+}, 5^{+}\right)$ & - & - & -5.0000000 & -3.89317903 & 5.98108858 \\
\hline$\left(1^{-}, 2^{+}, 3^{+}, 4^{+}, 5^{+}\right)$ & - & - & -5.0000000 & -16.3220021 & -10.3838132 \\
\hline$\left(1^{-}, 2^{-}, 3^{+}, 4^{+}, 5^{+}\right)$ & 12.50000 & 25.462469 & -1152.8431 & -4072.9383 & -3637.2496 \\
\hline$\left(1^{-}, 2^{+}, 3^{-}, 4^{+}, 5^{+}\right)$ & 12.50000 & 25.462469 & -6.1216296 & -90.221842 & -115.78367 \\
\hline
\end{tabular}

Table 1: Numerical results for all types of helicity configurations of the two-loop five-gluon amplitudes. For the amplitudes with vanishing trees, we set $\mathscr{A}^{(\text {norm })}=\mathscr{A}^{(1)}(\varepsilon=0)$, and for the MHV configurations $\mathscr{A}^{(\text {norm })}=\mathscr{A}^{(0)}$. Details are discussed in the text.

In table 1 we show our results for the helicity amplitudes. The results for amplitudes that vanish at tree-level have been normalized to $\mathscr{A}^{(\text {norm })}=\mathscr{A}^{(1)}(\varepsilon=0)$, that is to the corresponding (finite) one-loop amplitudes at order $\varepsilon^{0}$. The other amplitudes are normalized to the corresponding tree-level amplitudes. By this choice of normalization we remove any unphysical phase and expose more clearly the structure of the infrared poles. All infrared poles have been successfully checked against the expected results [42]. Furthermore, we have cross-checked against the known all-plus five-point results $[11,12,13,14]$ as well as validated the results of [17].

Obtaining the results in table 1 requires a large number of operations, including the sampling of a large number of trees and of the integrand function bases. This is performed over ten values of $D$ and three values of $D_{s}$ to reconstruct the dependence on the regulators. Although we have implemented structure for information caching, this nevertheless amounts to an under three-minute calculation for the most complex helicity structure in a single finite field with a single-threaded calculation performed on an i 7 Intel processor. We expect improvements in the efficiency of the calculation in the future, and we have also explored several parallelization strategies that can scale considerably computation times.

\section{Outlook}

We have presented the first calculation of a physically-relevant two-loop five-point amplitude with a full reduction to master integrals. The computation of the integral coefficients was achieved 
with the recently developed multi-loop numerical unitarity method, which exploits an integrand decomposition based on master integrands and surface terms. Importantly, it now feasible to assemble our amplitudes into squared matrix elements which can then be integrated over the physical phase space.

It is well known that unitarity-based techniques have good scaling properties as a function of the number of external legs (and also of the number of loops) making them very suitable for studying generic scattering amplitudes in field theories. While computing our results for four-point and five-point amplitudes, we observed that even at this early stage of the new developments, the computation of six-point two-loop amplitudes is within reach. Of course, these cases also pose the challenge of computing the corresponding master integrals, but we expect this to be possible in the near future.

Although gluon amplitudes are an excellent playground, we look forward to apply our techniques to more generic processes that can have interesting phenomenological applications. This includes processes with quarks, leptons and electro-weak bosons. Adding additional scales, for example by having external massive particles, poses only minor problems.

We have for the first time implemented an approach to reduce a full amplitude to master integrals employing finite field arithmetics. Such an approach holds several advantages, including the possibility of obtaining exact results for the integral coefficients when using rational phase-space points and exploiting integer-based operations for which modern CPUs are extremely efficient. For future applications to phenomenological calculations, we envisage a hybrid approach that makes use of both exact and floating point operations.

Another potential application of our exact integral coefficients is the exploration of the analytical structure of the amplitudes. Techniques for multivariate functional reconstruction have recently generated a lot of interest [36], which, when combined with fast amplitude evaluations, open a path towards obtaining analytic results for the amplitudes.

We have developed a computational framework for multi-loop numerical unitarity which we plan to make public to the wider high-energy physics community in the near future. We are planning to release the implementation in phases, starting with a version that gives access to the amplitudes that we have computed. Over time, we hope to provide more generic two-loop matrix elements that can be used for phenomenologically-relevant NNLO QCD computations.

\section{Acknowledgments}

We thank the organizers for the opportunity to present our work at this conference. The work of S.A., F.F.C., and B.P. is supported by the Alexander von Humboldt Foundation, in the framework of the Sofja Kovalevskaja Award 2014, endowed by the German Federal Ministry of Education and Research. The work of M.Z. is supported by the U.S. Department of Energy under Award Number DE-SC0009937.

\section{References}

[1] J. R. Andersen et al., Les Houches 2017: Physics at TeV Colliders Standard Model Working Group Report, in 10th Les Houches Workshop on Physics at TeV Colliders (PhysTeV 2017) Les Houches, 
France, June 5-23, 2017, 2018, 1803.07977 , http://lss.fnal.gov/archive/2018/conf/fermilab-conf-18-122-cd-t.pdf.

[2] F. R. Anger, F. Febres Cordero, H. Ita and V. Sotnikov, NLO QCD predictions for Wb $\bar{b}$ production in association with up to three light jets at the LHC, Phys. Rev. D97 (2018) 036018 [1712. 05721 ].

[3] ATLAS collaboration, M. Aaboud et al., Evidence for the $H \rightarrow b \bar{b}$ decay with the ATLAS detector, JHEP 12 (2017) 024 [1708.03299].

[4] CMS collaboration, A. M. Sirunyan et al., Evidence for the Higgs boson decay to a bottom quark-antiquark pair, Phys. Lett. B780 (2018) 501 [1709.07497].

[5] A. Gehrmann-De Ridder, T. Gehrmann and E. W. N. Glover, Antenna subtraction at NNLO, JHEP 09 (2005) 056 [hep-ph/ 0505111$].$

[6] J. Currie, E. W. N. Glover and S. Wells, Infrared Structure at NNLO Using Antenna Subtraction, JHEP 04 (2013) 066 [1301. 4693$].$

[7] M. Czakon, A novel subtraction scheme for double-real radiation at NNLO, Phys. Lett. B693 (2010) $259[1005.0274]$.

[8] S. Catani and M. Grazzini, An NNLO subtraction formalism in hadron collisions and its application to Higgs boson production at the LHC, Phys. Rev. Lett. 98 (2007) 222002 [hep-ph/ 0703012 ].

[9] R. Boughezal, X. Liu and F. Petriello, N-jettiness soft function at next-to-next-to-leading order, Phys. Rev. D91 (2015) 094035 [1504.02540].

[10] J. Gaunt, M. Stahlhofen, F. J. Tackmann and J. R. Walsh, N-jettiness Subtractions for NNLO QCD Calculations, JHEP 09 (2015) 058 [1505.04794].

[11] S. Badger, H. Frellesvig and Y. Zhang, A Two-Loop Five-Gluon Helicity Amplitude in QCD, JHEP 12 (2013) 045 [1310.1051].

[12] S. Badger, G. Mogull, A. Ochirov and D. O'Connell, A Complete Two-Loop, Five-Gluon Helicity Amplitude in Yang-Mills Theory, JHEP 10 (2015) 064 [1507.08797].

[13] T. Gehrmann, J. M. Henn and N. A. Lo Presti, Analytic form of the two-loop planar five-gluon all-plus-helicity amplitude in QCD, Phys. Rev. Lett. 116 (2016) 062001 [1511. 0540 9].

[14] D. C. Dunbar and W. B. Perkins, Two-loop five-point all plus helicity Yang-Mills amplitude, Phys. Rev. D93 (2016) 085029 [1603.07514].

[15] D. C. Dunbar, G. R. Jehu and W. B. Perkins, Two-loop six gluon all plus helicity amplitude, Phys. Rev. Lett. 117 (2016) 061602 [1605.06351].

[16] D. C. Dunbar, J. H. Godwin, G. R. Jehu and W. B. Perkins, Analytic all-plus gluon amplitudes in $Q C D, 1710.10071$.

[17] S. Badger, C. Brønnum-Hansen, H. B. Hartanto and T. Peraro, A first look at two-loop five-gluon scattering in QCD, 1712.02229.

[18] R. H. Boels, Q. Jin and H. Luo, Efficient integrand reduction for particles with spin, 1802.06761.

[19] H. A. Chawdhry, M. A. Lim and A. Mitov, Two-loop five-point massless QCD amplitudes within the IBP approach, 1805.09182.

[20] S. Abreu, F. Febres Cordero, H. Ita, M. Jaquier, B. Page and M. Zeng, Two-Loop Four-Gluon Amplitudes from Numerical Unitarity, Phys. Rev. Lett. 119 (2017) 142001 [1703. 05273]. 
[21] S. Abreu, F. Febres Cordero, H. Ita, B. Page and M. Zeng, Planar Two-Loop Five-Gluon Amplitudes from Numerical Unitarity, Phys. Rev. D97 (2018) 116014 [1712.03946].

[22] Z. Bern, L. J. Dixon, D. C. Dunbar and D. A. Kosower, One-loop n-point gauge theory amplitudes, unitarity and collinear limits, Nucl. Phys. B425 (1994) 217 [hep-ph/9403226].

[23] Z. Bern, L. J. Dixon, D. C. Dunbar and D. A. Kosower, Fusing gauge theory tree amplitudes into loop amplitudes, Nucl. Phys. B435 (1995) 59 [hep-ph/ 9409265$].$

[24] Z. Bern, L. J. Dixon and D. A. Kosower, One-loop amplitudes for $e^{+} e^{-}$to four partons, Nucl. Phys. B513 (1998) 3 [hep-ph/9708239].

[25] R. Britto, F. Cachazo and B. Feng, Generalized unitarity and one-loop amplitudes in N=4 super-Yang-Mills, Nucl. Phys. B725 (2005) 275 [hep-th/ 0412103 ].

[26] G. Ossola, C. G. Papadopoulos and R. Pittau, Reducing full one-loop amplitudes to scalar integrals at the integrand level, Nucl. Phys. B763 (2007) 147 [hep-ph/0609007].

[27] R. K. Ellis, W. T. Giele and Z. Kunszt, A Numerical Unitarity Formalism for Evaluating One-Loop Amplitudes, JHEP 03 (2008) 003 [0708.2398].

[28] W. T. Giele, Z. Kunszt and K. Melnikov, Full one-loop amplitudes from tree amplitudes, JHEP 04 (2008) 049 [0801.2237].

[29] C. F. Berger, Z. Bern, L. J. Dixon, F. Febres Cordero, D. Forde, H. Ita et al., An Automated Implementation of On-Shell Methods for One-Loop Amplitudes, Phys. Rev. D78 (2008) 036003 [0803.4180].

[30] S. Abreu, F. Febres Cordero, H. Ita, M. Jaquier and B. Page, Subleading Poles in the Numerical Unitarity Method at Two Loops, Phys. Rev. D95 (2017) 096011 [1703. 05255].

[31] H. Ita, Two-loop Integrand Decomposition into Master Integrals and Surface Terms, Phys. Rev. D94 (2016) 116015 [1510.05626].

[32] J. Gluza, K. Kajda and D. A. Kosower, Towards a Basis for Planar Two-Loop Integrals, Phys. Rev. D83 (2011) 045012 [1009.0472].

[33] W. Decker, G.-M. Greuel, G. Pfister and H. Schönemann, “SingulaR 4-1-0 - A computer algebra system for polynomial computations.” http://www.singular.uni-kl.de, 2016.

[34] D. A. Kosower, Direct Solution of Integration-by-Parts Systems, 1804.00131.

[35] A. von Manteuffel and R. M. Schabinger, A novel approach to integration by parts reduction, Phys. Lett. B744 (2015) 101 [1406.4513].

[36] T. Peraro, Scattering amplitudes over finite fields and multivariate functional reconstruction, JHEP 12 (2016) 030 [1608.01902].

[37] W. L. van Neerven and J. A. M. Vermaseren, LARGE LOOP INTEGRALS, Phys. Lett. 137B (1984) 241.

[38] G. 't Hooft and M. J. G. Veltman, Regularization and Renormalization of Gauge Fields, Nucl. Phys. B44 (1972) 189.

[39] C. G. Papadopoulos, D. Tommasini and C. Wever, The Pentabox Master Integrals with the Simplified Differential Equations approach, JHEP 04 (2016) 078 [1511.09404].

[40] T. Gehrmann and E. Remiddi, Two-loop master integrals for $\gamma * \rightarrow 3$ jets: The Planar topologies, Nucl. Phys. B601 (2001) 248 [hep-ph/ 0008287$].$ 
[41] J. Vollinga and S. Weinzierl, Numerical evaluation of multiple polylogarithms, Comput. Phys. Commun. 167 (2005) 177 [hep-ph/ 041025 9].

[42] S. Catani, The Singular behavior of QCD amplitudes at two loop order, Phys. Lett. B427 (1998) 161 [hep-ph/9802439]. 